Ferrata Storti Foundation

\title{
Dual cytoplasmic and nuclear localization of HTLV-1-encoded HBZ protein is a unique feature of adult T-cell leukemia
}

\section{Greta Forlani, ${ }^{1}$ Mariam Shallak, ${ }^{1}$ Alessandra Tedeschi, ${ }^{1}$ Ilaria Cavallari, ${ }^{2}$ Ambroise Marçais, ${ }^{3}$ Olivier Hermine ${ }^{3}$ and Roberto S. Accolla ${ }^{1}$}

${ }^{1}$ Laboratories of General Pathology and Immunology "Giovanna Tosi”, Department of Medicine and Surgery, University of Insubria, Varese, Italy; ${ }^{2}$ Istituto Oncologico Veneto IOV - IRCCS, Padua, Italy and ${ }^{3}$ Department of Hematology, Necker-Enfants Malades, University Hospital, Assistance Publique Hopitaux de Paris, Paris Descartes University, Paris, France

\section{ABSTRACT}

Volume 106(8):2076-2085

\section{Correspondence: \\ ROBERTO S. ACCOLLA \\ roberto.accolla@uninsubria.it}

Received: September 16, 2020 .

Accepted: January 19, 2021.

Pre-published: February 25, 2021.

https://doi.org/10.3324/haematol.2020.272468

(C)2021 Ferrata Storti Foundation

Material published in Haematologica is covered by copyright. All rights are reserved to the Ferrata Storti Foundation. Use of published material is allowed under the following terms and conditions:

https://creativecommons.org/licenses/by-nc/4.0/legalcode. Copies of published material are allowed for personal or internal use. Sharing published material for non-commercial purposes is subject to the following conditions:

https://creativecommons.org/licenses/by-nc/4.0/legalcode, sect. 3. Reproducing and sharing published material for commercial purposes is not allowed without permission in writing from the publisher.
$\Lambda$ dult T-cell leukemia-lymphoma (ATL), is a highly malignant T-cell neoplasm caused by human T-cell leukemia virus type 1 (HTLV-1), characterized by poor prognosis. Two viral proteins, Tax-1 and HTLV-1 basic-zipper factor (HBZ) play important roles in the pathogenesis of ATL. While Tax-1 can be found in both the cytoplasm and nucleus of HTLV-1 infected patients, HBZ is exclusively localized in the cytoplasm of HTLV-1 asymptomatic carriers and in patients with the chronic neurologic disease HTLV-I-associated myelopathy/tropical spastic paraparesis (HAM/TSP). HBZ is only localized in the nucleus of ATL cell lines, suggesting that the nuclear localization of HBZ can be a hallmark of neoplastic transformation. In order to clarify this crucial point, we investigated in detail the pattern of HBZ expression in ATL patients. We made use of our monoclonal antibody 4D4-F3, that at present is the only reported reagent, among the few described, able to detect endogenous HBZ by immunofluorescence and confocal microscopy in cells from asymptomatic carriers, HAM/TSP and ATL patients. We found that HBZ is localized both in the cytoplasm and nucleus of cells of ATL patients irrespective of their clinical status, with a strong preference for the cytoplasmic localization. Also Tax-1 is localized in both compartments. As HBZ is exclusively localized in the cytoplasm in asymptomatic carriers and in non-neoplastic pathologies, this finding shows that neoplastic transformation consequent to HTLV-1 infection is accompanied and associated with the capacity of HBZ to translocate to the nucleus, which suggests a role of cytoplasmic-to-nuclear translocation in HTLV-1mediated oncogenesis.

\section{Introduction}

Human T-cell leukemia virus type 1 (HTLV-1), the first identified human oncogenic retrovirus, is the etiological agent of a severe form of adult T-cell leukemia/lymphoma (ATL) ${ }^{1}$ and of HTLV-associated myelopathy/tropical spastic paraparesis (HAM/TSP), a progressive neurological disease.,3 10-20 million people are infected by HTLV-1 worldwide with a preferential localization in the Southwestern region of Japan, Australasia region, North and Central Africa, the Middle East, Central and South America and the Caribbean. ${ }^{4}$ Virus transmission occurs via cell-to-cell contact through breastfeeding, sexual intercourse, blood transfusions, and drug use with contaminated needles. ${ }^{5}$

Pathologies associated to HTLV-1 infections usually manifest after a long period of incubation that, in the case of ATL, may be of decades. ${ }^{6}$ ATL are divided into four clinical subtypes: acute, lymphoma, chronic, and smoldering, depending upon the relative lymphocytosis and lymphocyte abnormalities, the presence or absence of hypercalcemia, lactate dehydrogenase (LDH) serum concentration, and involvement of other organs. ${ }^{8}$

Although progress has been made in the pathophysiology and clinical treatment 
of the disease, prognosis of ATL remains poor., ${ }^{9,10}$ It is generally accepted that the oncogenic process leading to overt ATL proceeds through a multistep mode in which additional mutations are accumulated in the neoplastic clones. ${ }^{6,11,12}$ However, due to the specificity of ATL phenotype in comparison to other T- cell neoplasias, it is likely that virus infection and viral protein expression play a role in oncogenesis. ${ }^{13}$

Two viral proteins, the viral transactivator Tax-1 and the HTLV-1 basic-zipper factor (HBZ), have shown oncogenic activities in vitro and in vivo in experimental animal models. ${ }^{14,15}$ Tax-1 seems to be crucial in the onset of the oncogenic process mostly by disarranging several cellular activation pathways and particularly the NF- $\mathrm{BB}$ pathway. ${ }^{16-18}$ However, Tax-1 expression is lost in most of ATL cases suggesting that it might be dispensable for maintaining the neoplastic state. Loss of Tax-1 expression can be generated by both genetic lesions and epigenetic mechanisms. ${ }^{19,20}$

Onset of ATL clones with defects in Tax-1 expression are probably favored by immunological selection. Indeed, Tax-1 is strongly immunogenic and a preferential target of cytotoxic $T$ cells (CTL). ${ }^{21,22}$ In contrast to Tax- 1 , the negative strand-encoded $\mathrm{HBZ},{ }^{23}$ is expressed at all stages of infection and neoplastic transformation, ${ }^{24}$ suggesting that it may be required for the maintenance of the oncogenic process. Interestingly, it has been shown that not only HBZ protein but also $H B Z$ mRNA may be involved in the HTLV-1-mediated oncogenesis. ${ }^{25}$ Nevertheless, the intimate mechanism by which HBZ participates to the HTLV1-mediated neoplastic transformation still remains elusive. The subcellular distribution of HBZ in the various phases of the disease may be relevant as recent studies of our group demonstrated a peculiar and exclusive cytoplasmic distribution of HBZ protein in peripheral blood mononuclear cells (PBMC) of both asymptomatic carriers $(\mathrm{AC})^{26}$ and HAM/TSP patients ${ }^{27,28}$ compared to a preferential nuclear localization in a small sample of ATL cells. ${ }^{29}$

These findings were unprecedented and rather unexpected as previous studies, mostly performed in HBZ transfected cells, showed an exclusively nuclear distribution of the protein. . $^{30,31}$

Our studies, thus suggested that neoplastic transformation of HTLV-1-infected cells may be accompanied by a unidirectional displacement of HBZ from the cytoplasm to the nucleus.

Thus, we analyzed HBZ localization in fresh leukemic cells of ATL patients, representative of distinct clinical forms of the disease, to investigate potential HBZ transition from the cytoplasm to the nucleus and, in case of transition, to correlate it with distinct ATL clinical entities.

Here we show that leukemic cells from eight ATL patients can express HBZ not only in the nucleus but also in the cytoplasm. Neoplastic transformation is, thus accompanied by a dichotomy of HBZ localization and the exclusively cytoplasmic localization, as observed in AC and in HAM/TSP patients, is progressively modified to include nuclear localization of the protein.

\section{Methods}

\section{Cells and ethics statement}

This study was approved by the Ethical Committee (CPP Ile de FranceII, CNIL: number 1692254, registration number 000001072) and all surviving patients gave written informed consent. The study had a retrospective observational design. Patients with acute or chronic ATL at diagnosis were analyzed. See the Online Supplementary Appendix for further details.

\section{HTLV-1 proviral load measurement and determination of unspliced versus spliced HBZ mRNA}

Specific information on proviral load measurement and detection of unspliced versus spliced HBZ RNA is provided in the Online Supplementary Appendix.

\section{Cell treatments}

See the Online Supplementary Appendix for details.

\section{Immunofluorescence, flow cytometry and confocal microscopy}

Frozen vials containing PBMC were thawed by immediate passage from liquid nitrogen to a water bath set at $37^{\circ} \mathrm{C}$. Cells were washed with warm RPMI medium and immediately processed for immunofluorescence and flow cytometry analysis or for confocal microscopy as previsouly described. ${ }^{26,32,33}$ Additional information on antibodies used for detection of specific markers is reported in the Online Supplementary Appendix.

\section{Results}

\section{Cell surface phenotype and HBZ subcellular localization in acute adult T-cell leukemia-lymphoma patients: HBZ can reside in the cytoplasm}

We first investigated a group of four clinically defined acute ATL patients (namely PH131213, PH140126, PH160822 and PH1612N07).

Preliminary cell surface phenotype of PBMC from these patients showed that $\mathrm{CD} 4^{+} \mathrm{T}$ cells were the predominant, if not the total, cell subpopulation present in peripheral blood (Online Supplementary Figure S1). Interestingly, expression of the CD4 marker varied in the analyzed ATL patients, with strong (PH140126), moderate (PH131213 and $\mathrm{PH} 1612 \mathrm{~N} 07)$ and low (PH160822) expression. This, however, did not correlate with expression of the CD3 marker which was either very low or absent in PBMC of these patients, a result reminiscent of previous findings reported by our group and others. ${ }^{27,29,34,35}$ The T-cell activation marker CD25 was expressed in two (PH131213 and PH140126) of four patients' ATL cells, however, without correlation with the other T-cell activation marker, HLA class II. Expression and subcellular localization of HBZ was then analyzed by immunofluorescence and confocal microscopy Surprisingly, in contrast to what has been reported to date, in all four patients HBZ was predominantly found in the cytoplasm where it appeared as large dots (PH1612N07 and PH160822) with the tendency to converge in diffuse areas (PH131213 and PH140126) around the nucleus (Figure 1A). Interestingly, in PH1612N07 HBZ-positive cells showed the typical flowerlike phenotype described in acute ATL. HBZ cytoplasmic localization was confirmed by co-staining with the cytoplasmic marker vimentin and the nuclear marker DRAO5 (Figure 1B).

Of note, a wide variation in the percentage of $\mathrm{HBZ}$ positive cells was found in the four acute ATL patients, ranging from as low as $8.5 \%$ ( $\mathrm{PH} 131213$ ) to as high as $83 \%$ (PH140126) of PBMC leukemic cells (Online Supplementary Table S1). When present, HBZ nuclear localization was 
found in a minority of the HBZ-positive cells with the exception of patient PH140126 in which all HBZ-positive cells expressed the viral protein in both cellular compartments. Nevertheless, even in the case of PH140126, the nuclear localization was clearly under-represented with respect to the cytoplasmic localization (Figure 1A). In acute ATL, the proviral load (PVL) was generally higher than chronic ATL and did not strictly correlate with the percentage of HBZ-positive cells (Online Supplementary Table S1), even though the limited number of patients analyzed does not allow to draw a conclusion in terms of statistical significance. Interestingly, PVL values exceeded one copy of viral genome per cell in three of four patients, suggesting multiple integrations per single cell, as also demonstrated by recent molecular studies at a clonal level. ${ }^{36}$

In order to compare expression and subcellular localization of HBZ with those of Tax-1, a similar analysis was performed. Three of four acute ATL, namely PH140126, $\mathrm{PH} 1612 \mathrm{~N} 07$ and $\mathrm{PH} 160822$ showed expression of Tax-1 in $65 \%, 8,5 \%$ and $43 \%$ of cells, respectively, while
PH131213 ATL were negative for Tax-1 (Online Supplementary Table S1). It is interesting to note that the two patients expressing the highest number of Tax-1 positive cells (PH140126 and PH160822), were the same expressing the highest number of HBZ-positive cells.

As found for HBZ, Tax-1 subcellular localization was predominantly seen in the cytoplasmic compartment (Figure 2A and B; Online Supplementary Table S1). In assessable samples, most, but not all, Tax-1 positive cells were positive also for HBZ (Online Supplementary Table S1); in these cases HBZ and Tax-1 were mainly co-localized in the cytoplasmic compartment (Figure 2C).

\section{HBZ cytoplasmic localization is found also in chronic adult T-cell leukemia-lymphoma}

Four patients, clinically defined as chronic ATL, were analyzed. We first assessed the PBMC cell surface phenotype of these patients; as expected it was quite distinct from the phenotype observed in acute ATL. CD3 marker identifying all $\mathrm{T}$ cells, was clearly assessable in the majority of the cells in all patients analyzed with a noticeable

A
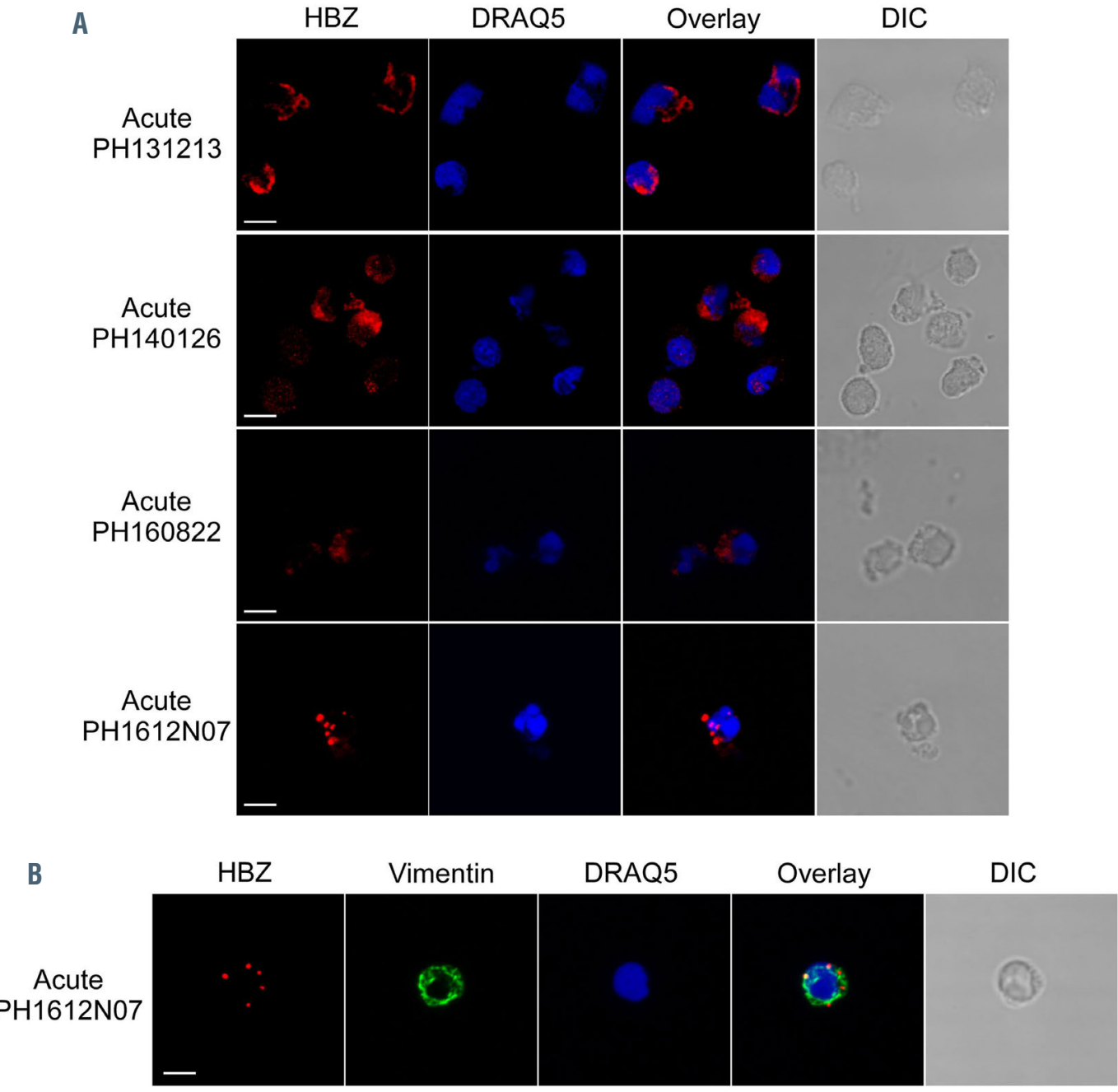

Figure 1. HBZ subcellular localization in peripheral blood mononuclear cells of patients with acute adult T-cell leukemia-lymphoma. (A) Peripheral blood mononuclear cells (PBMC) from PH131213, PH1401263, PH160822 and PH1612N07 patients were stained with the 4D4-F3 anti-HBZ monoclonal antibody (mAb) followed by Alexa Fluor 546-conjugated goat anti-mouse IgG1 antibody (red) and analyzed by confocal microscopy; DRAQ5 was used to detect the nucleus. (B). Specific counterstaining of cytoplasmic compartment in PH1612N07 patient's PBMC was performed by using anti-vimentin rabbit polyclonal antibody followed by goat anti-rabbit IgG conjugated to Alexa Fluor 488 (green). DIC represents the differential interference contrast image. At least 200 cells were analyzed. A representative image of HBZ staining is shown of each patient. All scale bars are $5 \mu \mathrm{m}$. 
distinction in patient PH171206 where only 20\% of the cells were CD3-positive (Online Supplementary Figure S2). Moreover, the CD4 T-cell marker identifying the T-helper subpopulation of CD3-positive T cells, although strongly expressed, was not present in the entire population (e.g., see patients $\mathrm{PH} 170706$ and $\mathrm{PH} 150610)$. Here, at variance with acute ATL, the observed phenotype was more similar to the CD4 phenotype observed in healthy donors PBMC. In chronic ATL patients the CD8 marker was expressed in a variable proportion of PBMC (Online Supplementary Figure S2), again mirroring the CD8 phenotype of normal PBMC more than the phenotype of acute ATL. The CD25 T-cell activation marker was not expressed, while HLA-DR was variably expressed, but always in a minor proportion of cells again mimicking the phenotype of normal PBMC.

Expression and subcellular localization of HBZ were then analyzed. As for acute ATL cases, HBZ was expressed in all cases ranging from $9.7 \%$ in patient $\mathrm{PH} 171206$ to $12.0 \%, 38.0 \%$ and $97.5 \%$ in patients $\mathrm{PH} 170706,150610$ and PH170918, respectively (Online Supplementary Table S1), demonstrating that the variability in the percentage of HBZ-positive cells was similar in chronic and acute ATL.
Thus, at least in the small number of cases analyzed, expression of HBZ may be not correlated with the clinical stage of the disease. Importantly, as observed in acute ATL, in all four chronic ATL cases HBZ was localized mostly in the cytoplasm (Figure 3A), with no cells expressing HBZ exclusively in the nucleus (Online Supplementary Table S1). Cells expressing nuclear HBZ were a minority as compared to the cells expressing HBZ only in the cytoplasm (29\% vs. $68 \%$ in $\mathrm{PH} 170918$, and $2.1 \%$ vs. $7.5 \%$ in $\mathrm{PH} 171206$ ). Moreover, PBMC of two chronic ATL patients, PH150610 and $\mathrm{PH} 170706$, did not show detectable nuclear HBZ expression at all (Figure 3A; Online Supplementary Table S1). $\mathrm{HBZ}$ appeared as distinctive dots, smaller in the nucleus when present as compared to the cytoplasm (Figure 3A, $\mathrm{PH} 170918$ and $\mathrm{PH} 171206)$ where the dots were often confluent creating a sort of ring around the nucleus (Figure 3A, $\mathrm{PH} 170706$ and $\mathrm{PH} 150610)$.

Interestingly Tax-1 was found expressed only in two of four chronic ATL patients, namely, PH170706 and PH171206, with a relative percentage of $6.4 \%$ and $1.0 \%$, respectively (Online Supplementary Table S1). Tax-1-positve cells of patients $\mathrm{PH} 170706$ expressed the viral marker only in the cytoplasm (Figure 3B; Online Supplementary Table S1)
A

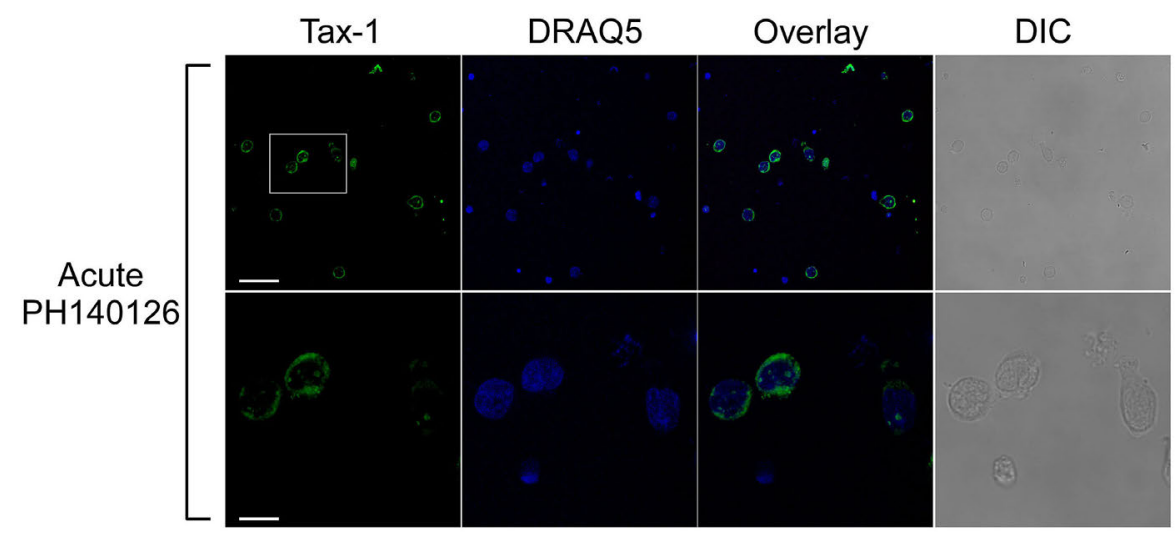

B

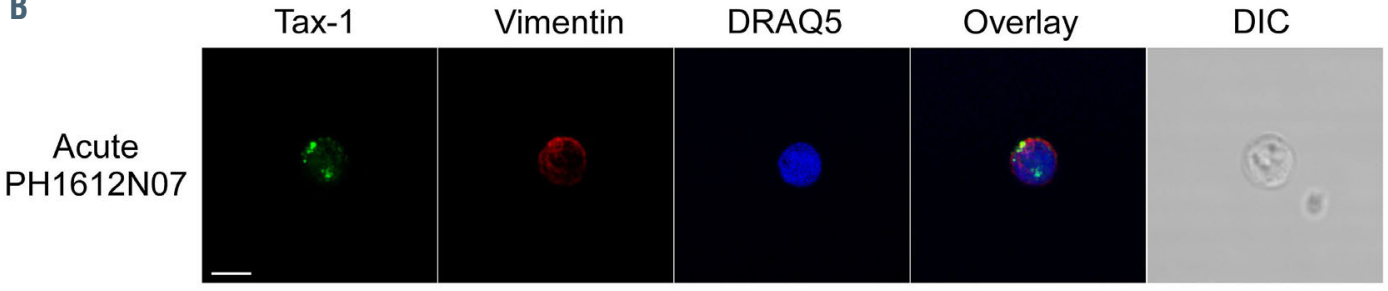

C

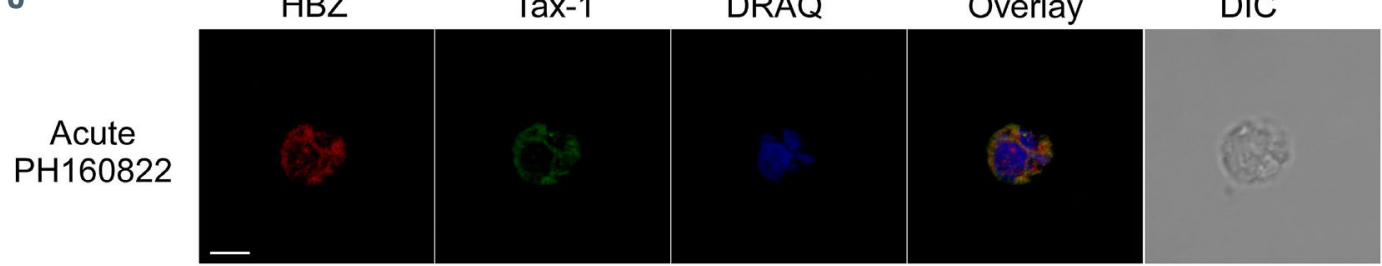

Figure 2. Tax-1 subcellular localization in peripheral blood mononuclear cells of patients with acute adult T-cell leukemia-lymphoma. (A) Peripheral blood mononuclear cells (PBMC) of representative acute adult T-cell leukemia-lymphoma patients PH40126 (A) and PH1612N07 (B) were stained with the A51-2 anti-Tax-1 monoclonal antibody $(\mathrm{mAb})$ followed by Alexa Fluor 488-conjugated goat-anti-mouse IgG2a antibody (green) and analyzed by confocal microscopy. Counterstaining of the nuclear or cytoplasmic compartments was performed by using DRAQ5 fluorescence probe to detect the nucleus (blue) and anti-vimentin rabbit polyclonal antibody followed by goat anti-rabbit IgG conjugated to Alexa Fluor 546 (B, red) to stain the cytoplasmic compartment. (C) PBMC of representative acute ATL patient PH160822 were costained with the 4D4-F3 anti-HBZ mAb followed by Alexa Fluor 546-conjugated goat anti-mouse IgG1 antibody (red) and with the A51-2 anti-Tax1 mAb followed by Alexa Fluor 488-conjugated goat-anti-mouse IgG2a antibody (green) and analyzed by confocal microscopy. DRAQ5 fluorescence probe was used to detect the nucleus (blue). DIC represents the differential interference contrast image. At least 300 cells were analyzed. All scale bars are $5 \mu m$. 
with a confluent dot-like fashion evenly distributed throughout this compartment; conversely Tax-1-positve cells of patients PH171206 expressed the viral marker both in the cytoplasm and the nucleus (Online Supplementary Table S1). Of note, Tax-1 was expressed in those two chronic ATL patients, PH170706 and PH171206, which expressed the lower percentage of HBZ positive cells. Moreover, as observed in acute ATL patients, not all Tax-1-positive cells were also HBZ-positive, as observed in PH170706 in which only $1 \%$ out of $6.4 \%$ of cells co-expressed the two viral proteins (Online Supplementary Table S1). As previously reported, ${ }^{26,27} \mathrm{HBZ}$ was expressed mostly in CD4-positive cells (Figure 4A). However in PBMC of those patients with a distinctive and relatively normal proportion of CD8-positive cells, such as PH170918 (see the Online Supplementary Figure S1), HBZ expression could also be found in few CD8positive cells (Figure 4B).

\section{Predominance of spliced versus unspliced form of $H B Z$} mRNA in adult T-cell leukemia-Iymphoma

Then we verified whether predominant HBZ cytoplasmic localization in leukemic cells correlated with preferen- tial expression of one of the two described forms of $H B Z$ mRNA, spliced versus unspliced. ${ }^{37,38}$ We found a clear predominance of the spliced versus unspliced HBZ form in our leukemic samples without appreciable differences between acute and chronic ATL (Online Supplementary Figure S3). This was in line with the results obtained in HAM/TSP patients and asymptomatic carriers, which expressed HBZ exclusively in the cytoplasm (Online Supplementary Figure S3), ${ }^{26,27}$ but at variance with the ATL2 leukemic cell line and with $\mathrm{PH} 1505$ patient leukemic cells, which expressed mostly nuclear HBZ. ${ }^{27}$ Here the unpliced form was predominant (ATL-2) or similar (PH1504) to spliced form.

\section{Cytoplasmic HBZ protein does not shuttle between the cytoplasm and nucleus}

We have previously shown that in PBMC from both asymptomatic carriers and HAM/TSP patients the exclusive cytoplasmic localization of HBZ could not be modified by treatment with Leptomycin B (LMB), a specific inhibitor of CRM1/exportin-mediated nuclear export, ${ }^{39}$ strongly suggesting that in AC and HAM/TSP, HBZ does

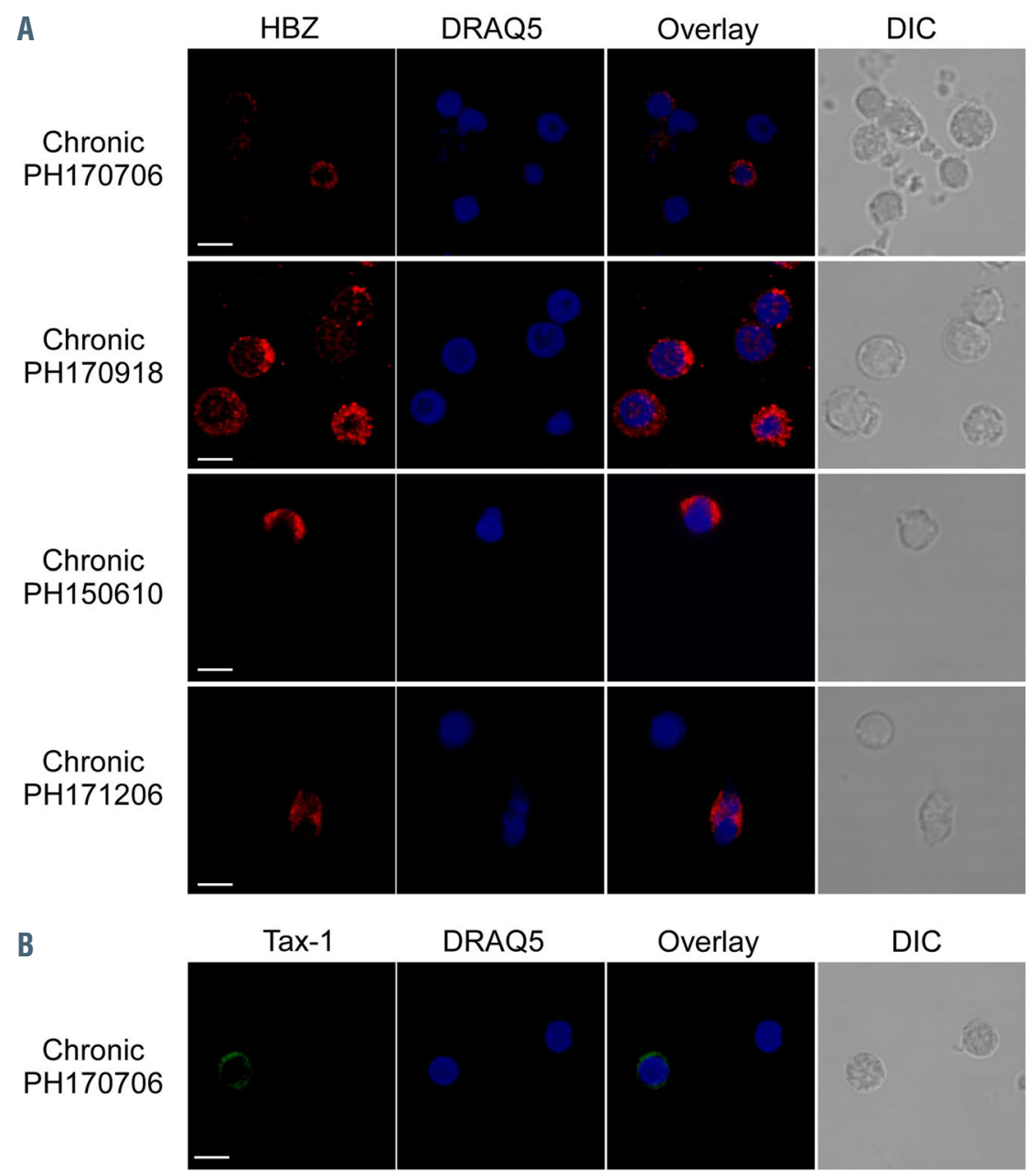

Figure 3. HBZ subcellular localization in peripheral blood mononuclear cells of patients with chronic adult T-cell leukemia-lymphoma. (A) Peripheral blood mononuclear cells (PBMC) of representative chromic leukemia patients PH150610, PH170706, PH170918 and PH171206 were stained with the 4D4-F3 anti-HBZ monoclonal antibody (mAb) followed by Alexa Fluor 546-conjugated goat anti-mouse lgG1 antibody (red) and analyzed by confocal microscopy. DRAQ5 fluorescence probe was used to detect the nucleus. (B) PBMC of representative chronic leukemia patient PH170706 was stained with the A51-2 anti-Tax-1 mAb followed by Alexa Fluor 488-conjugated goat-anti-mouse IgG2a antibody (green) and analyzed by confocal microscopy. Nucleus was stained with DRAQ5. DIC represents the differential interference contrast image. At least 300 cells were analyzed. One representative image of HBZ staining derived from PBMC samples of each patient is shown. At least 300 cells were analyzed. All scale bars are $5 \mu \mathrm{m}$. 
not shuttle between the cytoplasm and nucleus. ${ }^{27}$ Since in acute and chronic ATL, HBZ could be localized in both compartments, with a clear preference for the cytoplasm, it was important to assess whether the entire pool of cytoplasmic HBZ could indeed shuttle between cytoplasm and nucleus. Cells were treated with LMB and analyzed by immunofluorescence and confocal microscopy. Results presented in Figure 5A show that this was not the case because $\mathrm{LMB}$ treatment did not result in the accumulation of HBZ protein in the nucleus in either acute (PH140126) or chronic (PH170918) ATL cells (Figure 5A).

As a control, LMB treatment of normal PHA-treated $\mathrm{PBMC}$ was instead capable to significantly retain the p65/RelA component of the NF- $\kappa B$ complex in the nucleus (Figure $5 \mathrm{~B}$ ). Taken together, these results strongly suggest that the cytoplasmic component of HBZ protein in both acute and chronic ATL does not ostensibly shuttle between the cytoplasm and nucleus through CRM1/exportin regulated mechanism.

Calreticulin may regulate Tax-1 nuclear export by interacting with, and retaining the viral protein in the cytoplasm in a concentration dependent fashion. ${ }^{40}$ In order to verify whether calreticulin might contribute to the cytoplasmic localization of HBZ, we costained PBMC from both acute and chronic patients with $\mathrm{HBZ}$ and calreticulin specific antibodies. Cytoplasmic HBZ did not colocalize with calreticulin. Moreover, we could not detect a significant increased expression of calreticulin in HBZ-positive cells, compared to HBZ-negative cells (Online Supplementary Figure S4A). Cytoplasmic Tax-1 instead par- tially colocalized with calreticulin in acute ATL patient PH160822, although also in this case we could not observe a clear different level of calreticulin expression in Tax-1 positive cells compared to Tax-1-negative cells (Online Supplementary Figure S4B)

\section{The cytoplasmic localization of HBZ in adult T-cell leukemia-lymphoma patients may partially affect p65/RelA but not JunD subcellular distribution}

Previous studies have shown that HBZ inhibits Tax-1mediated NF- $\kappa B$ activation and consequent transcription of various NF- $\mathrm{B}$ target genes by targeting p65/RelA suggesting that this can contribute to the attenuation of viral replication and persistence of infection. ${ }^{41,42}$ Here we analyzed the subcellular localization of p65/RelA in PBMC isolated from both acute and chronic ATL subjects. Interestingly, we found p65/RelA in the cytoplasm of HBZ-positive cells of both acute and chronic patients, partially colocalizing with HBZ (Online Supplementary Figure S5A, PH160822 and $\mathrm{PH} 170706$, respectively). This suggests that a possible, although limited, HBZ-p65/RelA cytoplasmic interaction may limit p65/RelA transition into the nucleus and the activation of the NF- $\kappa \mathrm{B}$ target genes.

In order to evaluate the impact of the observed cytoplasmic localization of HBZ on other cellular pathways targeted by the viral protein, we analyzed the subcellular distribution of the transcription factor JunD, previously shown to weakly interact and partially colocalize with HBZ in an ATL patient expressing HBZ in the nucleus. ${ }^{29}$ When HBZ was expressed in the cytoplasm, JunD was still localized

A

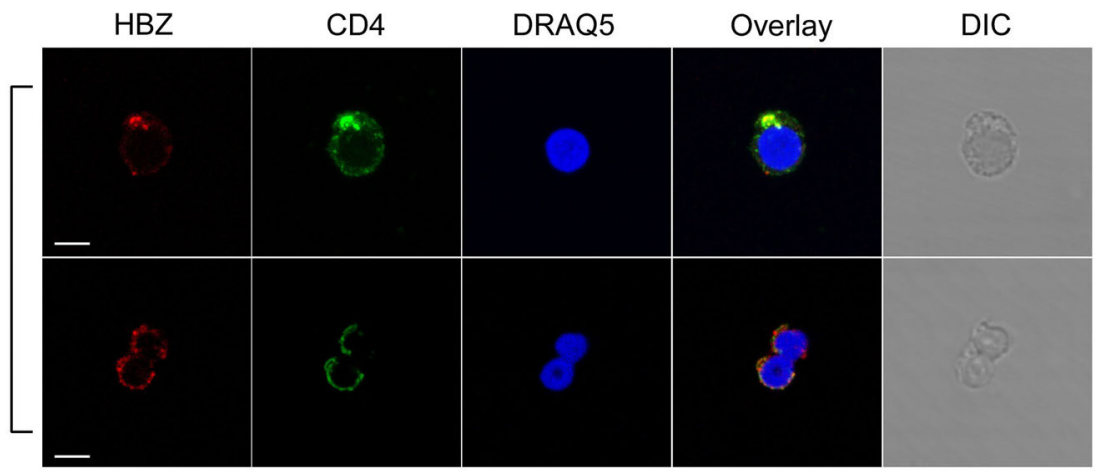

B

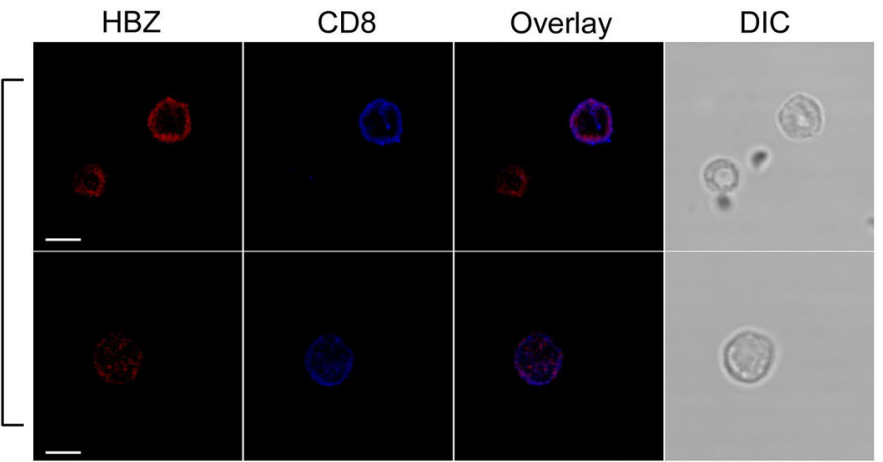

Figure 4. HBZ expression in T-cell subpopulations of chronic adult T-cell leukemia-lymphoma. Confocal microscopy analysis of peripheral blood mononuclear cells (PBMC) from representative chronic (CH) leukemic PH170918. (A) Upper panels: PBMC of patient CH PH170918 were costained with the 4D4-F3 anti-HBZ monoclonal anitbody (mAb) followed by Alexa Fluor 546-conjugated goat anti-mouse IgG1 antibody (red) and with the anti-CD4 rabbit mAb (RabmAb) followed by Alexa Fluor 488-conjugated goat-anti-rabbit IgG antibody (green). Nucleus was stained with DRAQ5. (B) PBMC of CH patient PH170918 were costained with the 4D4-F3 anti-HBZ mAb followed by Alexa Fluor 546-conjugated goat anti-mouse IgG1 antibody (red) and with the anti-CD8 RabmAb directly conjugated to Alexa Fluor 647 (blue). At least 200 cells were analyzed; DIC represents the differential interference contrast image. representative images derived from each sample are shown. All scale bars are $5 \mu \mathrm{m}$. 
in the nucleus (Online Supplementary Figure S5B, PH160822 and $\mathrm{PH} 170706)$.

\section{Discussion}

The nuclear localization of HBZ in ATL has been largely inferred from studies describing the many functions that this protein exerts directly on cellular gene promoters of infected cells or by interacting with a large number of host transcription factors in the nucleus. ${ }^{6,43-46}$ Recent investigations conducted by confocal microscopy and biochemical approaches focused on endogenous HBZ protein were consistent with the idea that leukemic cells from HTLV-1infected ATL patients express the viral protein in the nucleus. ${ }^{29}$ These findings, in association with elegant studies in HBZ-transgenic animal models, ${ }^{15}$ gave strong support to the idea that HBZ nuclear localization is instrumental for, or at least strongly associated with, the maintenance of the leukemic state. On the other hand, more recent and precise immune localization studies have clearly indicated that HBZ expression is always and only confined to the cytoplasm in HTLV-1 AC and in HAM/TSP patients in contrast to Tax-1 localization that can be found in both compartments. ${ }^{26,27}$ Since AC and more rarely HAM/TSP states may evolve to the leukemic state, it could be, thus, realistic to think that HBZ translocation from the cytoplasm to the nucleus is associated with the genesis of the neoplastic process leading to ATL. In order to verify this hypothesis it was instrumental to demonstrate that stages of leukemic transformation could indeed show evidence of cytoplasmic-only or cytoplasmicnuclear localization of endogenous HBZ protein. In this study, we have demonstrated that indeed leukemic cells from ATL patients could be found that express HBZ in the cytoplasm with high frequency. Apparently, therefore, we filled the gap with previous studies and showed the unidi- rectional subcellular translocation from the cytoplasm to the nucleus of HBZ during oncogenic transformation. Moreover, HBZ was expressed not only in $\mathrm{CD}^{+} \mathrm{T}$ cells, the preferential target of the HTLV-1 retrovirus, but also in a very small percentage of $\mathrm{CD}^{+} \mathrm{T}$ cells indicating that $\mathrm{CD}^{+} \mathrm{T}$ cells can be infected by HTLV-1, confirming our previous observations, ${ }^{26,27}$ and persist in ATL patients.

Several findings reported in this study require further investiagtions to be framed in the context of unidirectional cytoplasm-to-nucleus localization of HBZ in ATL.

A rather unexpected and important finding was that none of the eight patients, either acute or chronic ATL, analyzed in this study had cells expressing HBZ exclusively in the nucleus. As a partial explanation, we can adduce that most of the previous studies describing endogenous HBZ localization were carried out in leukemic cell lines possibly representative of highly selected phenotypes in culture, while only three patients were described with an exclusive nuclear localization by a similar confocal microscopy approach. ${ }^{27,29}$ In this context, it is important to stress that previously analyzed ATL patients and those described in this study did not differ in terms of geographic origin or ethnic group being all afro caribbeans but one ( $\mathrm{PH} 150610)$.

A second important finding relates to the evidence, again common to both acute and chronic ATL, that in cells expressing HBZ in both the cytoplasm and nucleus, the cytoplasmic fraction was always consistently higher than the nuclear fraction. Actually, in two ATL chronic patient (PH150610 and PH170706), HBZ was only observed in the cytoplasm. A relative exception was represented by the acute ATL patient PH140126 displaying the highest percentage of HBZ-positive cells (83\%) all of which were positive for both cytoplasmic and nuclear localization. The unprecedented HBZ cytoplasmic localization in leukemic patients found in this study was paralleled by the known more abundant expression of the spliced versus

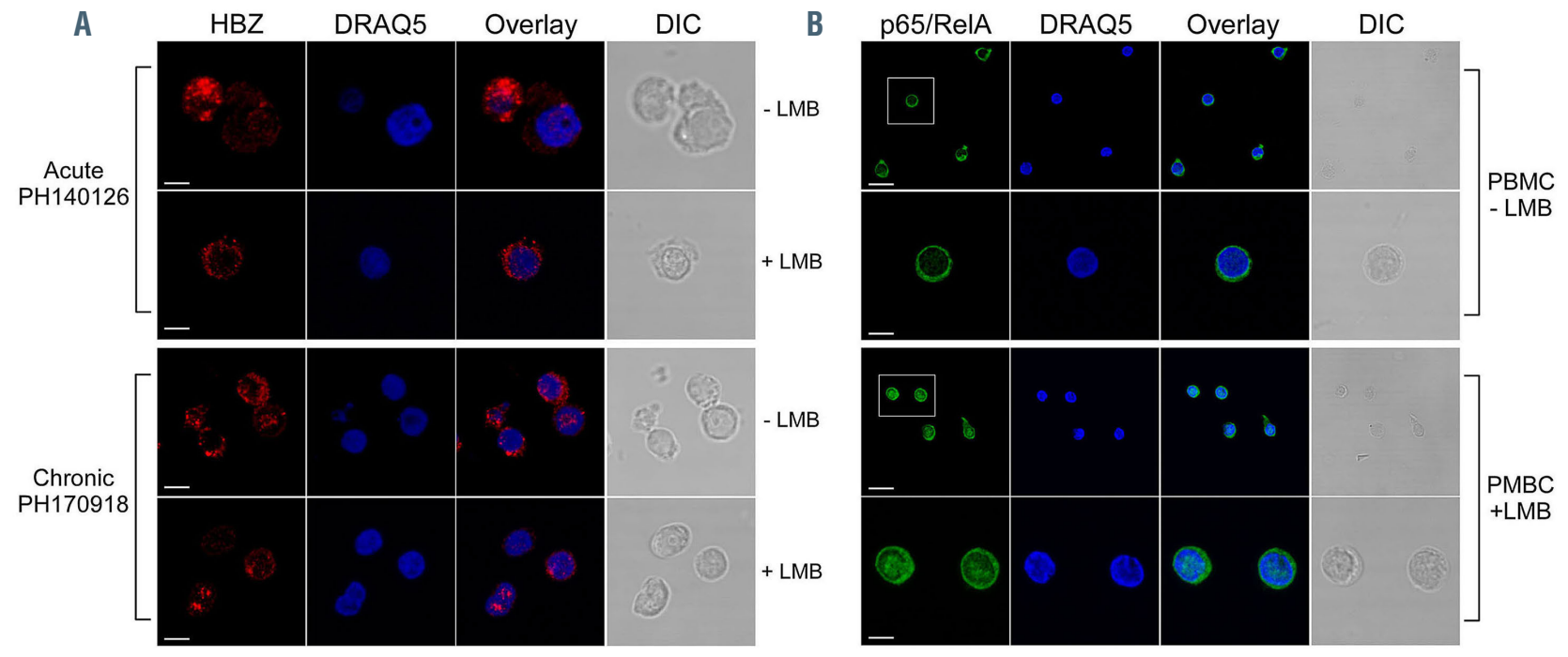

Figure 5. Cytoplasmic HBZ is retained in this compartment and does not shuttle to the nucleus. (A) Peripheral blood mononuclear cells (PBMC) of representative acute $\mathrm{PH} 140126$ and chronic $\mathrm{PH} 170918$, were either treated (+LMB, bottom panels) or not treated (-LMB, top panels) with Leptomycin B (LMB), an inhibitor CRM1/exportin-mediated nuclear export, before fixing and stained with the anti-HBZ 4D4-F3 monoclonal antibody (mAb) followed by Alexa Fluor 546-conjugated goat anti-mouse IgG1 antibody (red) and analyzed by confocal microscopy. (B) As control of inhibition of nuclear export by LMB, treated (+LMB) and untreated (-LMB) cells were fixed and stained with antibodies against p65/RelA followed by Alexa Fluor 546-conjugated goat-anti-rabbit secondary antibody (red) and analyzed by confocal microscopy. One representative image on the field and an enlargement of this are shown. Nucleus was stained with DRAO5 (blue). DIC represents the differential interference contrast image. One representative image is shown for each sample. At least 300 cells were analyzed. All scale bars are $5 \mu \mathrm{m}$. 
unspliced form of $H B Z$ mRNA, ${ }^{25}$ corroborated also in HAM/TSP patients and in AC that have exclusive HBZ protein cytoplasmic localization. ${ }^{26,27}$ Conversely, the ATL2 leukemic cell line and leukemic cells of a previously described patient (PH1505), displaying a predominant HBZ nuclear localization, showed a more abundant or similar unspliced versus spliced $H B Z$, suggesting a correlation between subcellular protein localization and distinct forms of alternatively spliced $H B Z$ mRNA.

Taken together, these results show for the first time that the dual cytoplasmic/nuclear localization of HBZ is an exclusive feature of ATL, giving further credit to the hypothesis of subcellular re-localization of HBZ in leukemogenesis (Figure 6).

The high percentage of HBZ-positive cells observed in chronic ATL, a situation usually characterized by a low number of leukemic cells ${ }^{47}$ as well as the exclusive cytoplasmic localization of HBZ found in two chronic ATL patients, remain to be clarified. Are the described conditions the mirror of a specific step of the neoplastic process such as the anticipation of an acute phase of the disease or a phase in which HBZ is progressively relocated into the nucleus without any modification of the clinical status, or, provocatively, the manifestation of novel disease subgroups? The first hypothesis seems unlikely as clinical follow-up suggests that these patients have not evolved toward an aggressive phenotype, indicating that other factors, possibly involved in the cytoplasmic dislocation of HBZ, are needed to mark the transition versus a different clinical state of ATL.

Previous studies of our group have clearly demonstrated that both in asymptomatic carriers and HAM/TSP patients the exclusive HBZ cytoplasmic localization was due to a retention in this subcellular compartment, as inhibition of CRM-1 dependent nuclear export by LMB did not result in nuclear retention of the viral protein. ${ }^{26,27}$ It was therefore important to assess whether in cells of ATL patients showing both cytoplasmic and nuclear localization, HBZ could freely shuttle between the two subcellular compartments. This was neither the case in acute nor chronic ATL, suggesting, besides the above described association of spliced-cytoplasmic versus unspliced-nucleus, the existence of an active mechanism of cytoplasmic HBZ retention even in presence of a quota of nuclear protein, and irrespective of the clinical form of the disease (Figure 6). One possible candidate for the cytoplasmic retention of HBZ could be calreticulin previously shown to regulate Tax-1 nuclear export. ${ }^{40}$ However, at least for the patients analyzed, HBZ was not clearly confined in a calreticulin subcellular compartment, while Tax-1 partially colocalized with calreticulin. A more in-depth analysis with an increased number of acute and chronic ATL subjects might further clarify this aspect.

Recent results have suggested that HBZ can be retained in the cytoplasm by interacting with the T cellspecific molecule THEMIS. ${ }^{48}$ Conversely, we showed that this did not explain the cytoplasmic retention of HBZ in cells of HAM/TSP patients. ${ }^{26}$ Thus, although in ATL a partial involvement of THEMIS in the retention of HBZ in the cytoplasm cannot be excluded, it is certainly important to further investigate the possibility that different cytoplasmic anchoring molecules or molecular complexes may also be involved in the cytoplasmic localization of HBZ in ATL. Whatever the mechanism, it is

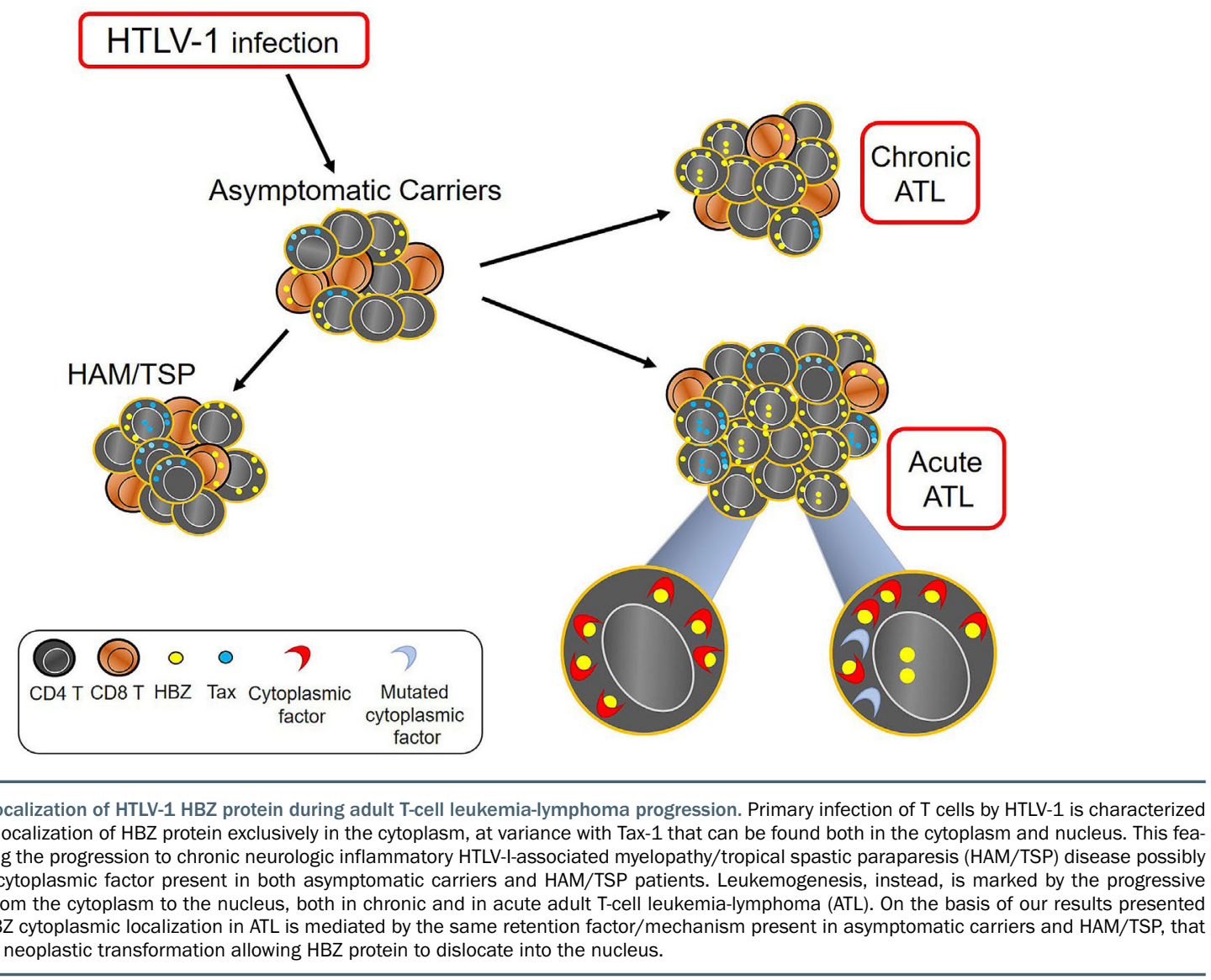


clear that a quota of the HBZ molecular pool can translocate into the nucleus and can be retained there to functionally participate in the maintenance of the leukemic phenotype. The molecular basis of this nuclear retention remains to be investigated.

An additional relevant aspect of the present study was the expression and subcellular localization of Tax-1 protein. Tax-1 was not expressed in three of eight patients analyzed in this study, confirming previous findings on the silencing of the viral oncogene in ATL. ${ }^{19}$ Interestingly, however, a clear difference was observed between acute and chronic ATL, as Tax-1 was expressed in three of four acute ATL in a relevant proportion of cells, ranging from $8.4 \%$ to $65.0 \%$ of PBMC, and only in two of four chronic ATL but in a considerably low proportion of cells, namely $1.0 \%$ and $6.4 \%$ of PBMC. This finding correlated quite well with the cell surface phenotype of leukemic cells in that PBMC of chronic ATL patients resembled more closely the phenotype observed in healthy individuals.

An exclusive Tax-1 nuclear localization was found in very few cases and only in acute ATL, whereas the vast majority of the Tax-1-positive cells expressed the viral protein both in the cytoplasm and nucleus. The fact that in acute ATL cases analyzed in this study Tax-1 was expressed in a considerable proportion of cells, although unexpected, may indeed support the idea that increased and/or sustained cell proliferation observed in the acute leukemic state is associated with a burst of Tax-1 expression, as recently proposed. ${ }^{49,50}$ Furthermore, both in acute and in chronic ATL most of Tax-1-positive cells were also HBZ-positive suggesting a distinct behaviour with respect to what has been recently found in asymptomatic carriers and HAM/TSP patients in which HBZ and Tax-1 were very rarely found co-expressed in the same cells. ${ }^{26,27}$ As stressed above, this may be correlated with the distinct replicative state of the cells in asymptomatic carriers and HAM/TSP patients as compared to leukemic patients.

In conclusion, the results presented in this study establish for the first time a correlation between modification of the subcellular localization of HBZ and acquisition of neoplastic state in subjects infected by HTLV-1. Future studies will be devoted to the clarification of the intimate molecular mechanisms that are at the basis of the cytoplasmic and nuclear retention of HBZ in HTLV-1-mediated leukemogenesis.

\section{Disclosures \\ No conlicts of interest to disclose.}

\section{Contributions}

GF and RSA conceived the experiments, performed data analysis, wrote and revised the manuscript; GF, MS, AT and IC performed the experiments; $A M$ and $O H$ provided ATL samples and reviewed the manuscript.

\section{Funding}

This study was supported by the University of Insubria "FAR 2018 and 2019" (to RSA and GF); by The Institutional Grant 2017, University of Insubria (to RSA); in part by the European Community FP7 Grant no. 602893 "Cancer Vaccine Development for Hepatocellular Carcinoma-HepaVAC" http://www.hepavac.eu (to RSA, and GF); by grants from the Institut National du Cancer (INCA), France (to $\mathrm{OH}$ and FM).

\section{References}

1. Poiesz BJ, Ruscetti FW, Gazdar AF, Bunn PA, Minna JD, Gallo RC. Detection and isolation of type $C$ retrovirus particles from fresh and cultured lymphocytes of a patient with cutaneous T-cell lymphoma. Proc Natl Acad Sci U S A. 1980;77(12):7415-7419.

2. Gessain A, Vernant JC, Maurs L, et al. Antibodies to human T-lymphotropic virus type-1 in patients with tropical spastic paraparesis Lancet. 1985;326(8452):407-410.

3. Osame M, Usuku K, Izumo S, et al. HTLV-I associated myelopathy, a new clinical entity. Lancet. 1986;327(8488):1031-1032.

4. Gessain A, Cassar O. Epidemiological aspects and world distribution of HTLV-1 infection. Front Microbiol. 2012;3:388-388.

5. Pique C, Jones KS. Pathways of cell-cell transmission of HTLV-1. Front Microbiol. 2012;3:378.

6. Watanabe T. Adult T-cell leukemia: molecular basis for clonal expansion and transformation of HTLV-1-infected T cells. Blood. 2017;129(9):1071-1081.

7. Uchiyama T, Yodoi J, Sagawa K, Takatsuki K, Uchino H. Adult T-cell leukemia: clinical and hematologic features of 16 cases. Blood. 1977;50(3):481-492.

8. Shimoyama M, Group moTLS. Diagnostic criteria and classification of clinical subtypes of adult T-cell leukaemia-lymphoma. Br J Haematol. 1991;79(3):428-437.

9. Hermine O, Ramos JC, Tobinai K. A review of new findings in adult T-cell leukemialymphoma: a focus on current and emerging treatment strategies. Adv Ther. 2018;35(2):135-152.
10. Cook LB, Fuji S, Hermine O, et al. Revised Adult T-cell Leukemia-Lymphoma International Consensus Meeting Report. J Clin Oncol. 2019;37(8):677-687

11. Kataoka K, Nagata Y, Kitanaka A, et al. Integrated molecular analysis of adult $\mathrm{T}$ cell leukemia/lymphoma. Nat Genet. 2015;47(11):1304-1315.

12. Rosewick N, Durkin K, Artesi M, et al. Cisperturbation of cancer drivers by the HTLV$1 / B L V$ proviruses is an early determinant of leukemogenesis. Nat Commun. 2017;8:15264.

13. Matsuoka M, Jeang K-T. Human T-cell leukaemia virus type 1 (HTLV-1) infectivity and cellular transformation. Nat Rev Cancer. 2007;7(4):270-280.

14. Hasegawa H, Sawa H, Lewis MJ, et al. Thymus-derived leukemia-lymphoma in mice transgenic for the Tax gene of human T-lymphotropic virus type I. Nat Med. 2006;12(4):466-472.

15. Satou Y, Yasunaga J-i, Zhao T, et al. HTLV-1 bZIP factor induces T-cell lymphoma and systemic inflammation in vivo. PLoS Pathog. 2011;7(2):e1001274.

16. Tanaka A, Takahashi C, Yamaoka S, Nosaka T, Maki M, Hatanaka M. Oncogenic transformation by the tax gene of human T-cell leukemia virus type I in vitro. Proc Natl Acad Sci U S A. 1990;87(3):1071-1075.

17. Grassmann R, Berchtold S, Radant I, et al. Role of human T-cell leukemia virus type 1 $\mathrm{X}$ region proteins in immortalization of primary human lymphocytes in culture. J Virol. 1992;66(7):4570-4575.

18. Grassmann R, Aboud M, Jeang K-T. Molecular mechanisms of cellular transformation by HTLV-1 Tax. Oncogene.
2005;24(39):5976-5985.

19. Takeda S, Maeda M, Morikawa S, et al. Genetic and epigenetic inactivation of tax gene in adult T-cell leukemia cells. Int J Cancer. 2004;109(4):559-567.

20. Taniguchi Y, Nosaka K, Yasunaga J-i, et al. Silencing of human T-cell leukemia virus type I gene transcription by epigenetic mechanisms. Retrovirology. 2005;2:64.

21. Jacobson S, Shida H, McFarlin DE, Fauci AS, Koenig S. Circulating CD8+ cytotoxic T lymphocytes specific for HTLV-I pX in patients with HTLV-I associated neurological disease. Nature. 1990;348(6298):245248.

22. Kannagi M, Harada S, Maruyama I, et al. Predominant recognition of human $\mathrm{T}$ cell leukemia virus type I (HTLV-I) pX gene products by human CD8+ cytotoxic T cells directed against HTLV-I-infected cells. Int Immunol. 1991;3(8):761-767.

23. Gaudray G, Gachon F, Basbous J, BiardPiechaczyk M, Devaux C, Mesnard J-M. The complementary strand of the human Tcell leukemia virus type 1 RNA genome encodes a bZIP transcription factor that down-regulates viral transcription. J Virol. 2002;76(24):12813-12822.

24. Usui T, Yanagihara K, Tsukasaki K, et al. Characteristic expression of HTLV-1 basic zipper factor (HBZ) transcripts in HTLV-1 provirus-positive cells. Retrovirology. 2008;5:34.

25. Satou Y, Yasunaga J-i, Yoshida M, Matsuoka M. HTLV-I basic leucine zipper factor gene mRNA supports proliferation of adult $\mathrm{T}$ cell leukemia cells. Proc Natl Acad Sci U S A. 2006;103(3):720-725.

26. Forlani G, Baratella M, Tedeschi A, Pique C, 
Jacobson S, Accolla RS. HTLV-1 HBZ protein resides exclusively in the cytoplasm of infected cells in asymptomatic carriers and HAM/TSP patients. Front Microbiol. 2019;10:819.

27. Baratella M, Forlani G, Raval GU, et al. Cytoplasmic localization of HTLV-1 HBZ protein: a biomarker of HTLV-1-associated myelopathy/tropical spastic paraparesis (HAM/TSP). PLoS Negl Trop Dis. 2017;11(1):e0005285.

28. Baratella M, Forlani G, Accolla RS. HTLV-1 HBZ viral protein: a key player in HTLV-1 mediated diseases. Front Microbiol. 2017;8:2615.

29. Raval GU, Bidoia C, Forlani G, Tosi G, Gessain A, Accolla RS. Localization, quantification and interaction with host factors of endogenous HTLV-1 HBZ protein in infected cells and ATL. Retrovirology. 2015;12:59.

30. Hivin P, Frédéric $M$, Arpin-André $C$, et al. Nuclear localization of HTLV-I bZIP factor (HBZ) is mediated by three distinct motifs. J Cell Sci. 2005;118(7):1355-1362.

31. Hivin P, Basbous J, Raymond F, et al. The HBZ-SP1 isoform of human T-cell leukemia virus type I represses JunB activity by sequestration into nuclear bodies. Retrovirology. 2007;4:14.

32. Tosi G, Forlani G, Andresen V, et al. Major histocompatibility complex class II transactivator CIITA is a viral restriction factor that targets human T-cell lymphotropic virus type 1 Tax-1 function and inhibits viral replication. J Virol. 2011;85(20):10719-10729.

33. Forlani G, Abdallah R, Accolla RS, Tosi G. The major histocompatibility complex class II transactivator CIITA inhibits the persistent activation of NF- $\mathrm{KB}$ by the human T cell lymphotropic virus type 1 Tax-1 oncoprotein. J Virol. 2016;90(7):3708-3721.

34. Shirono K, Hattori T Matsuoka M Matsushita S, Asou N, Takatsuki K. Adult T cell leukemia cell lines that originated from primary leukemic clones also had a defect of expression of CD3-T cell receptor complex. Leukemia. 1988;2(11):728-733.

35. Suzushima H, Asou N, Hattori T, Takatsuk $\mathrm{K}$. Adult T-cell leukemia derived from S100 beta positive double-negative (CD4- CD8-) T cells. Leuk Lymphoma 1994:13(3-4):257-262.

36. Artesi M, Marçais A, Durkin K, et al. Monitoring molecular response in adult Tcell leukemia by high-throughput sequencing analysis of HTLV-1 clonality. Leukemia. 2017;31(11):2532-2535.

37. Cavanagh $\mathrm{MH}$, Landry S, Audet B, et al. HTLV-I antisense transcripts initiating in the 3'LTR are alternatively spliced and polyadenylated. Retrovirology. 2006;3:15.

38. Murata K, Hayashibara T, Sugahara K, et al. A novel alternative splicing isoform of human T-cell leukemia virus type $1 \mathrm{bZIP}$ factor (HBZ-SI) targets distinct subnuclear localization. J Virol. 2006;80(5):2495-2505.

39. Ossareh-Nazari B, Bachelerie F, Dargemont C. Evidence for a role of CRM1 in signalmediated nuclear protein export. Science. 1997:278(5335):141-144.

40. Alefantis T, Flaig KE, Wigdahl B, Jain P. Interaction of HTLV-1 Tax protein with calreticulin: implications for Tax nuclear export and secretion. Biomed Pharmacother. 2007;61(4):194-200.

41. Zhao T, Yasunaga J, Satou Y, et al. Human Icell leukemia virus type $1 \mathrm{bZIP}$ factor selectively suppresses the classical pathway of NF-kappaB. Blood. 2009;113(12):2755-2764.
42. Zhi H, Yang L, Kuo YL, Ho YK, Shih HM, Giam CZ. NF-kB hyper-activation by HTLV1 tax induces cellular senescence, but can be alleviated by the viral anti-sense protein HBZ. PLoS Pathog. 2011;7(4):e1002025.

43. Yamagishi M, Fujikawa D, Watanabe $T$, Uchimaru K. HTLV-1-mediated epigenetic pathway to adult T-cell leukemia-lymphoma. Front Microbiol. 2018;9:1686.

44. Ma G, Yasunaga J-I, Matsuoka M Multifaceted functions and roles of HBZ in HTLV-1 pathogenesis. Retrovirology. 2016;13:16.

45. Giam C-Z, Semmes OJ. HTLV-1 Infection and adult T-cell leukemia/lymphoma - a tale of two proteins: Tax and HBZ. Viruses. 2016;8(6):161.

46. Matsuoka M, Mesnard J-M. HTLV-1 bZIP factor: the key viral gene for pathogenesis. Retrovirology. 2020;17(1):2.

47. Bazarbachi A, Suarez F, Fields P, Hermine O. How I treat adult T-cell leukemia/lymphoma. Blood. 2011;118(7):1736-1745.

48. Kinosada $H$, Yasunaga J-I, Shimura $K$, et al. HTLV-1 bZIP factor enhances T-cell proliferation by impeding the suppressive signaling of co-inhibitory receptors. PLoS Pathog. 2017;13(1):e1006120

49. Mahgoub M, Yasunaga J-I, Iwami S, et al Sporadic on/off switching of HTLV-1 Tax expression is crucial to maintain the whole population of virus-induced leukemic cells. Proc Natl Acad Sci U S A. 2018;115(6) e1269-e1278.

50. Billman MR, Rueda D, Bangham CRM Single-cell heterogeneity and cell-cycle-related viral gene bursts in the human leukaemia virus HTLV-1. Wellcome Open Res. 2017 $2 \cdot 87$ 